\title{
Slope Inspection System: Using image processed by machine learning algorithm to determine risk of slope failure
}

\author{
S. H. Mat Shukorl, S. B. Abdul Hamid ${ }^{1}$, N. Kasim ${ }^{2}$, Z. Ab. Moin ${ }^{3}$ \\ ${ }^{I}$ International Islamic University Malaysia, Faculty of Engineering, Department of Mechatronics Engineering, \\ Jalan Gombak, Kuala Lumpur, Malaysia, 53100 \\ ${ }^{2}$ International Islamic University Malaysia, Faculty of Engineering, Department of Civil Engineering, Jalan \\ Gombak, Kuala Lumpur, Malaysia, 53100 \\ ${ }^{3}$ Ministry of Higher Education Malaysia, Department of Polytechnic and Community Education, Galeria PJH, \\ Persiaran Perdana, Putrajaya, Malaysia, 62100 \\ *Email: syamsul_bahrin@iium.edu.my
}

\begin{abstract}
Malaysia is a tropical country that experiences rainy and hot weather throughout the year. The higher rainfall intensity leads to higher landslide occurrences in Malaysia. Landslides that occur nearby human settlements increase the risk and hazard to the public and properties that lead to significant economic losses. There are various methods in surveying the risk and hazard of landslide areas such as terrestrial laser scanning (TLS) and global positioning system (GPS). Most of the past research uses the conventional method which requires an in-situ field survey, lab analysis, and an additional software package to determine the hazard level for a slope. The conventional method is inefficient and time-consuming. In this paper, the potential of a machine-learning algorithm to improve the conventional approach in detecting the hazard in a landslide is discussed. The algorithm assesses the level of risk based on trained supervised images identified by experts in the field. Using the trained network model, it was found that Convolution Neural Network (CNN) can perform better than Fully Connected Layer with reduced processing time for sampled images at an accuracy of $66 \%$ compared and 33\% respectively. However, when the trained CNN is subjected to actual IIUM slope images of which all have been identified as low risk by local expert, the actual accuracy of the network reduced to $50 \%$, of which the remaining are predicted as high risk. More training data could be added to the CNN to improve the current accuracy.
\end{abstract}

Indexed Terms- Landslide, Deep Learning, Slope Failure, Machine Learning, Python, Big Data.

\section{INTRODUCTION}

Malaysia is in a tropical region closed to the equator and is experiencing dry and humid conditions with a lot of rainfall throughout the years [1]. The rain or monsoon season commonly occur in two periods, which are (November-March) and (May-September) each year. This natural phenomenon has frequently resulted in two major disasters in Malaysia, knowingly flood and landslides. Each of these calamities severely affected the country, especially in terms of the socio-economy, and safety of the people. Flood generally occurs sporadically during heavy rainfall, due to the overflow of surface runoff that accumulate high amount of water within a short period of time and/or the presence of poor drainage system. Meanwhile, landslide is a failure that could occur on a sloping landform. Mass movement or landslide is a major natural hazard that may happen in mountainous region worldwide. In addition, according to data from the US National Aeronautics Space Administrations (NASA), Malaysia has recorded 171 landslides between 2007 and March 2016 [2] making it the $10^{\text {th }}$ highest landslide occurrences in the world.

There are several factors that contribute to the incident of landslides in Malaysia such as geological, morphological, hydrogeological factors and due to the poor slope management [3]. Other contributing 
factors including abusive prescriptive method, inadequate study of past failure, design related issues such as insufficient site-specific ground investigation, increasing development of highland or hilly terrains and hills projects in the pipeline. Understanding the characteristics of the slope and a way to monitor a condition that can lead to the landslide is important as it would help to reduce the risk of landslides. All these approaches can be integrated as a mechanism of an early warning system, where the relevant authorities can take the pre-emptive measures to alert the people regarding the upcoming disaster. Hence, the losses from both disasters could be mitigated and save people lives from any casualties.

There are different ways that could be performed to monitor and identify the risk of failure in a slope that could cause a landslide. Afeni and Cawood [4] mentioned that the use of manual total station instruments in slope monitoring is widely applicable for most studies. Other than that, Hou [5] conducted a slope monitoring work by utilizing Global Positioning System (GPS) associated with few sensor instruments to identify the ground parameters (ground displacement, ground water level). In this paper, an automated system using machine learning algorithm is proposed. This will reduce the need for human to perform inspection or surveying in-situ on the hill or high-risk slope areas. Using images captured during maintenance or other activities, the images could be processed accordingly and the risk level can be identified for further administrative action. Thus, a remote sensing and monitoring could be implemented using this methodology. Furthermore, problem of poor management and insufficient investigation of the site could be reduced. However, in order for the automated surveillance system to be implemented effectively, it needs to be highly accurate and reliable. In this paper, red green blue (RGB) images of slopes will be analyzed using a deep learning algorithm and the risk associated to the slope will be identified. Two different types of deep learning techniques will be proposed and compared - fully connected layer and convolution neural network. The accuracy of the prediction from the training data and testing data will be evaluated and analyzed.

\section{CURRENT TECHNIQUE OF SLOPE MONITORING}

In order to implement the automated slope monitoring system, different machine learning algorithm is discussed and introduced. However, before the technique is discussed and the proposed solution is mention, it is important that the basis behind slope failure is discussed, along with the current inspection system.

\subsection{Slope Inspection System}

There are various inspection techniques that have been developed to improve the slope inspection.

\subsubsection{Mobile Robot}

Ultrasonic sensor module (USM) Model HC-SR04, have been used as a low-cost solution to detect slope. In this case, two USM installed at different height to perform slope detection and calculation [6]. Measuring the inclination and declination angle from identified distance, the USM sensor is able to identify a positive or negative slope. However, the system has its own limitation. Due to underlying principle of reflection in ultrasonic detection, not all types of slopes are suitable to be measured using this technique. As line of sight is usually required for an ultrasonic sensor, certain type of slope causes the ultrasonic reflection to be reflected away from the detector sensor. In addition, since the robot is moving on the ground for slope detection, certain angle such as high slope level could not be detected by the system. Furthermore, the system requires the surveying robot to reach the surveying field to perform the desired inspection. This causes two main challenges in implementation. One, it takes time to reach the needed destination, and two the robot may need to move through or around an obstacle such as rock, tree and drain before arriving to its final sensing destination. 


\subsubsection{Unmanned Aerial Vehicle (UAV)}

Unmanned Aerial Vehicle (UAV) technology or drones in recent years have led engineer to develop a new approach in determining the risk of a slope. Being low in weight and increasing low in cost, drone usage has shown an increase in the field of architecture, engineering and construction monitoring [7]. Some of the more advance drones nowadays are fully equipped with smart construction monitoring and reporting system. In addition, drones are also increasing in popularity due to their affordability and their capability of carrying high resolution cameras and other remote sensing equipment [8].

\subsubsection{Terrestrial Laser Scanner}

Terrestrial laser scanner has been use to survey the development of rock fall frequency-magnitude relationship on slopes [9]. Information sensed by laser scanner could be utilized to assign hazard rating, prioritizing inspection work and to develop a hazard management and mitigation systems. Comparing the sequential scans by terrestrial laser scanner, the possibility of a rock fall events and the rock fall source locations and event volume can be identified and calculated.

\subsubsection{Synthetic Aperture Radar (SAR)}

Synthetic Aperture Radar (SAR) is a coherent active microwave remote sensing instruments, which are capable to efficiently map the scattering properties of the earth's surface [10]. Generally, SAR is used in geodesy and remote sensing. In addition, SAR could measure quantitatively, the earth deformation due to natural hazard such as deterioration of buildings and public facilities [10].

\subsubsection{Infrared Thermography}

Infrared Thermography is a non-contact technique which is used to generate a surface temperature profile of the test area in the form of grey scale or colored infrared images. Infrared thermography is one of the techniques that can be used in predictive and proactive maintenance.

\subsubsection{Digital photogrammetry.}

Other methods for landslide mapping or ground based technologies in slope analysis are digital photogrammetry (DP). DP is a technique to acquire dense 3D geometric information for real world objects from stereoscopic image. The technique has shown to have extensive applications in a variety of field. There are variety of software that had been used such as PhotoScan, PhotoModeler and Leica Photogrammetry Suite (LPS).

\subsection{Processing image using machine learning technique}

Machine learning algorithm depends on an inventory data set. This data set is used for training and validation process [11]. Thus, it is crucial that access to an accurate landslide inventory data set at all stages monitoring; modelling and mapping landslide risk is available [12]. There are four methods of machine learning techniques that can be utilized to analyze the slope: artificial neural network (ANN), support vector machines (SVM), random forest (RF) and deep learning convolution neural network (CNN) [11].

Meezaal has shown that recurrent Neural Network (RNN) and Multilayer perceptron neural network (MLP-NN) using textural features can be utilized for landslide detection [13], [14]. The techniques typically require an input data source like ortophotos or Lidar-derived data. In addition, the comparison between a maximum likelihood ratio (MLR) and (ANN) as automatic landslide detection using multispectral advance space borne thermal emission and reflection radiometer (ASTER) images was studied [15].

In addition, Zhu et al [16] and Ghorbanzadeh [12] have shown that convolution neural network $(\mathrm{CNN})$ is able to achieve good results in various image analyses. CNN has also been utilized in very 
high resolution (VHR) image classification and segmentation, semantic segmentation and scene annotation using different high spatial resolution, aerial images and object detection. Most of the work in $\mathrm{CNN}$ involved detecting an object, in particular searching for distinct characteristics of the objects and finally classify an object into different categories. The classification algorithm depends heavily on large number of labelled images for object recognition.

In this paper, we are trying to apply CNN on RGB images that had been captured by cameras, perform needed analysis and to predict the risk of the slopes.

\section{DEVELOPMENT OF MACHINE LEARNING ALGORITHMS}

\subsection{Landslide Hazard and Risk Assessment}

Landslide hazard is a process to identify the level of hazard at the landslide prone-area, while the risk assessment is a technique to manage the landslide risk in terms of the probability of land sliding to occur, runout behavior of landslide debris, vulnerability of property and people to landslide, landslide risk to property and people, and management strategies and decision-making [17]. Numerous research have addressed the approach of minimizing the landslide hazard and carrying the risk assessment study in respective region as an effort to mitigate the losses from the mass movement [17], [18]. Jamaluddin and Hussein [18] stated the landslide hazard and risk assessment is an important tool in addressing uncertainty inherent in the occurrence of slope failure. The social and economic losses due to the landslide can be reduced by an effective management and planning. This can be accomplished throughout hazard and risk assessment study. In this paper, different images of slopes need to be classified into respective categories which are high, medium and low risk for both training and testing data set to be used by machine learning algorithm. Chow and Mohamad [19] have developed a landslide assessment that is based on large assessment for land use planning. This assessment is able to classify the level of hazard according to its categories.

This study will perform the slope analysis using parameters of the weightage of slope gradient and geomorphological unit, by referring to [19]. According to Chow and Mohamad, there are four attributes that are being used in terrain analysis and classification maps which are slope gradient, morphology, activity and erosion, and instability [19]. Table 1 and Table 2 show the weightage of slope gradient and geomorphological unit based on study conducted by Chow and Mohamad [19]. The hazard score, $H_{s}$ can be calculated using Equation (1) [20], which is based on the weightage category identified in Table 1 and Table 2.

$$
\operatorname{Hazard}, H_{s}=\frac{W_{c}+W_{g}}{1.8708}
$$

where $W_{c}$ is the weightage of the slope class, which is based on the slope angle in Table 1 , and $W_{g}$ is the weightage of the geomorphological unit, which is based on water content as shown in Table 2.

Table 1: Information value weightage for slope classes [20]

\begin{tabular}{|c|c|c|}
\hline $\begin{array}{c}\text { Slope } \\
\text { classes }\end{array}$ & Slope range & $\begin{array}{c}\text { Information value } \\
\text { weightage, } \boldsymbol{W}_{\boldsymbol{c}}\end{array}$ \\
\hline 1 & $0-10$ degrees & -0.3563 \\
\hline 2 & $10-20$ degrees & 0.6225 \\
\hline 3 & $20-30$ degrees & 0.3024 \\
\hline 4 & $30-40$ degrees & 0.6064 \\
\hline 5 & $40-50$ degrees & 0.6096 \\
\hline 6 & $50-60$ degrees & 0.8093 \\
\hline 7 & $>60$ degrees & 1.63 \\
\hline
\end{tabular}


Table 2: Information values weightage for geomorphological unit [20].

\begin{tabular}{|c|c|}
\hline $\begin{array}{c}\text { Geomorphological } \\
\text { unit }\end{array}$ & $\begin{array}{c}\text { Information value } \\
\text { weightage, } \boldsymbol{W}_{\boldsymbol{g}}\end{array}$ \\
\hline Alluvial plain & -0.9875 \\
\hline Water body & 0.3734 \\
\hline River/fluvial terrace & -1.9232 \\
\hline Valley fill & -0.8116 \\
\hline Residual hill & 0.2408 \\
\hline Blocky hill & 0.6477 \\
\hline Scarp & 2.3586 \\
\hline Top Hill & 2.1556 \\
\hline
\end{tabular}

In addition, Chow et al [19] have also categorized and rate the risk of landslide hazard based on the rating low to very high as shown in Table 3. Furthermore, weightage for consequences based on the type of risk and land use/premise could also be seen in Table 4 . The total risk score, $R_{s}$ could then be calculated using the Equation (2) [19].

Table 3: Rate of landslide hazard according to its classification [19].

\begin{tabular}{|c|c|c|}
\hline Class & Hazard rating & Hazard score, $\boldsymbol{H}_{\boldsymbol{s}}$ \\
\hline 1 & Low & $<0.25$ \\
\hline 2 & Moderate & $0.26-0.5$ \\
\hline 3 & High & $0.51-0.75$ \\
\hline 4 & Very High & $>0.76$ \\
\hline
\end{tabular}

Total Risk, $R_{S}=H_{S} C_{S}$

Table 4: Weightage for consequential score [19]

\begin{tabular}{|c|c|c|}
\hline Type of risk & Land use / premises & $\begin{array}{c}\text { Consequences } \\
\text { score, } C_{s}\end{array}$ \\
\hline \multirow[t]{7}{*}{ Risk of lives } & Critical buildings affected & 20 \\
\hline & Normal buildings affected & 10 \\
\hline & Isolated building affected & 5 \\
\hline & Very busy trunk road & 10 \\
\hline & Busy trunk road & 7 \\
\hline & Moderately used trunk road & 5 \\
\hline & Seldom used trunk road & 1 \\
\hline \multirow{7}{*}{$\begin{array}{l}\text { Economic } \\
\text { loss }\end{array}$} & Damage to farm/park & 3 \\
\hline & Business area (only access) & 10 \\
\hline & Only access to housing area & 6 \\
\hline & Temp. diversion (> 1 day) & 3 \\
\hline & Temp. diversion ( $\leq 1$ day $)$ & 0 \\
\hline & Alternative road ( $\geq 5 \mathrm{~km})$ & 3 \\
\hline & Alternative road $(<5 \mathrm{~km})$ & 0 \\
\hline \multirow{2}{*}{$\begin{array}{l}\text { Public } \\
\text { Utilities }\end{array}$} & Affected & 10 \\
\hline & Not affected & 0 \\
\hline \multirow{5}{*}{$\begin{array}{l}\text { Proximity of } \\
\text { building to } \\
\text { suspected } \\
\text { landslide }\end{array}$} & Very close & 10 \\
\hline & Close & 5 \\
\hline & Possibly affected & 2 \\
\hline & Unlikely to be affected & 0 \\
\hline & Not affected & 0 \\
\hline
\end{tabular}


The risk rating could then be identified based upon the mapping of the total risk score, $R_{s}$ and the risk rating as shown in Table 5.

Table 5: Classification of landslide risk rating used [19]

\begin{tabular}{|c|c|}
\hline Risk Rating & $\begin{array}{c}\text { Total risk score, } \\
\boldsymbol{R}_{\boldsymbol{s}}\end{array}$ \\
\hline Low risk & $<12.5$ \\
\hline Moderate risk & $12.6-25$ \\
\hline High risk & $>26$ \\
\hline
\end{tabular}

With this, the rating for each image could be identified and categorized accordingly based on low, moderate or high risk. Each training image and data set will be analyzed using equation (2) in order to identify the valid data that will be used to train the machine learning algorithm.

\subsection{Comparison of Different Machine Learning Algorithm}

Convolution Neural Network is a feed neural network that is utilized in image recognition and image classification. CNN consist of 4 layers which are convolution layer, ReLU layer, Max Pooling layer, and Fully Connected layer. Fig. 1 [21] shows CNN process as a whole. Convolution operation is the basis in convolution neural network. In CNN, every image is represented in the form of arrays of pixel values as shown on the left side of Fig. 1. The bird image is being convolve with grains or filter and the result is call convolve feature.

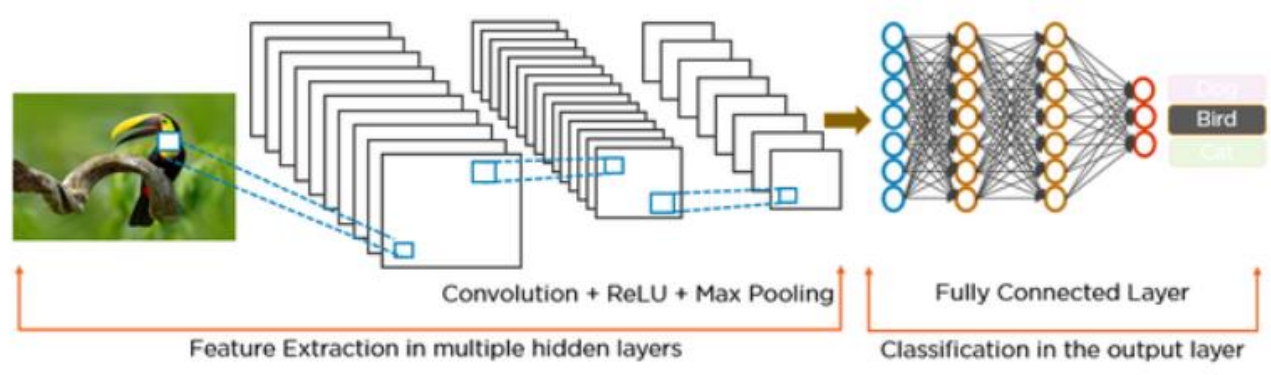

Figure 1: Process of convolution neural network [21]

Once the convolution feature map was extracted, the next step is to move them to an activation function, ReLU layer. The output of ReLU layer is called rectified feature map. The rectified feature map now goes through a max pooling layer. Max pooling method simplifies the different information from the rectified feature map and creates a pooled feature map. Pooling layers uses different filters to identify different parts of the images like edges, corners, body, hand, eyes and leg. Next, the pooled feature map goes through flattening process and finally to fully connected layer.

Fully connected layer model alone without convolution technique can be used to perform prediction and classification, but there are limitations. Fully connected layer and convolution neural network are similar since both methods learn by changing weights and biases. The input from both networks is going through neurons, perform a dot product and follow up with an activation function like ReLU (Rectified Linear Unit).

However, the main problem of fully connected layer is slow training time and have higher chances of overfitting due to large numbers of parameter. For example, in order to classify images with size 64 x 64 x 3, fully connected layers need 12288 weights in the first hidden layer. Hence, the number of weights will be even bigger when the size image is $225 \times 225 \times 3$. For convolution neural network, the main image matrix is reduced to a matrix of lower dimension in the first layer in a process called convolution. A comparison between convolution neural network and fully connected layer will be discussed later in the results and discussion. 


\subsection{Machine Learning Algorithm Implementation}

\subsubsection{Machine learning application and Implementation}

There are different applications that could be utilized to implement the machine learning algorithm. In this paper, Python and Jupyter Notebook is used to implement the deep learning algorithm. Python programming language consist of tools and libraries that related with machine learning and data science. The most common libraries are Numpy and Matplotlib. Numpy is a library for scientific computing, perfect for multivariable calculus and linear algebra. In addition, Numpy library provide array class which can be compared to Python list that can be created as vector of matrix. To perform data visualization, Matplotlib library is used to create the 2D and 3D plots. The Jupyter Notebook is an opensource web application that allows developer to create and share documents that contain live code, equations, visualizations and narrative text.

In addition, there are two important frameworks in building and training models for deep learning which are Tensorflow and Keras. Keras is available as a built-in within Python, thus generally is more user friendly that Tensorflow. However, Tensorflow provides both high level and low-level application program interface, compared to Keras which only provides high-level application interface. In terms of flexibility, Tensorflow's execution allows for immediate iteration along with intuitive debugging. Keras on the other hand, offer simple and consistent high-level application program interface and follows best practices to reduce cognitive load for the users.

There are three types of machine learning method - supervised, unsupervised and reinforcement learning. Since the technique involved a local expert, a supervised approach is chosen to supervise the prediction of the slope by the machine learning algorithm. Supervised learning requires dataset consist of example in an input-output pairs. Each pair consists of data sample that used to make prediction and expected outcome called label as shown on the Fig. 2, of which network model are developed and modified based on the error feedback.

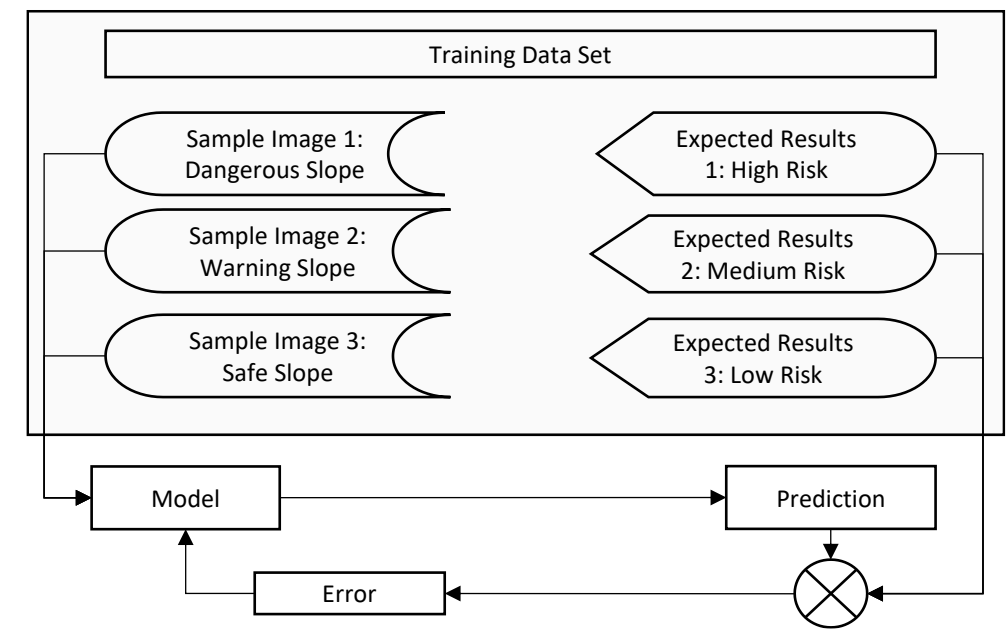

Figure 2: Diagram on Supervised Learning Utilized to Predict Slope Risk in this project.

\subsubsection{Acquisition of Data for Machine Learning}

There are two types of data that will be use in this paper which is testing data and training data. For training data, the image data must be as many as possible to achieve high accuracy in classifying the risk of the slope from the study area. The training and testing data set had been collected from various resources such as images from internet, books and journals known as public data sets and images captured around Genting Sempah, Pahang, Malaysia known and private data set. Images from steep of the slope in the trunk road around Genting Sempah are included in the training and testing dataset as 
the area is reported to be prone to landslide. The images resolution utilized in the training and validation varies from 224 pixels by 224 pixels to 1200 pixels by 900 pixels. The training dataset consist of 328 images, of which this data has been further stratified by internal expert as having to belong into three categories which are low, medium, and high risk. The training and testing data are split into a Train/Test ratio of 85:15 for low and high risk, and 70:30 for medium risk respectively. The main reason for the different ratio between low-high and medium, is to ensure that medium risk has a higher sampling test rate compared to low and medium.

Focus work area for this project is around the Gombak Campus of the International Islamic University Malaysia and the surrounding hilly areas. There are multiple cases of flash flood that happen around this university and the worse is in 2014 that result on five cars belonging to lecturers and students were submerged in a meter of water because of heavy downpour. Previously, it was mentioned that heavy and sudden rainfall is one of the factors that lead to landslide. As the soil moisture increases, the risk of landslide increases. With a total of more than 18 thousand students in the campus during semester running, it is important that landslide detection is performed to avoid additional landslide occurrences that can cost life and damages to facilities.

\section{RESULTS AND DISCUSSION}

\subsection{Implementation of Machine Learning Algorithm}

The process in constructing the algorithm of deep learning can be seen in Fig. 3. The first step is to import the necessary library. The libraries are Tensorflow, Numpy, Matplotlib, Keras, os and sys. The next step is to execute a directory call or dataset files from computer into the algorithm to pre-process the data. Pre-process of data or data manipulation involved altering the image data set such as its size and to increase the amount of data. By increasing the amount of data, overfitting error in training the deep learning algorithm can be effectively reduced.

The value of each pixel in an array then need to flatten, before inserting them into to the model algorithm, to enable the neural network to learn the key features from the image. Two model algorithms were designed in order to identify which model provides a higher accuracy in predicting the hazard for a particular slope. The accuracy and loss curves were plotted to identify, any overfitting in both of the models. Figure 4 show the accuracy curves and loss curves for a) fully connected layer and b) convolution neural network model.

From Fig. 4 (a) and (b), it could be observed that both figures experience overfitting issues but fully connected layer much worst as there are huge gaps between validation accuracy and training accuracy after epochs of 10. On the contrary, although the loss curves and accuracy curves of convolution neural network contain noise and not smooth but the trend still decreasing and increasing respectively. The noise within the CNN may be due to the different image types within the data set, i.e., the public and private data set.

The main problems of fully connected layer are slow in training time and higher chances of overfitting error due to large number of input parameters into model algorithm. In comparison, the main image matrix of convolution neural network model id reduced to a matrix of lower dimension in the first layer through convolution process before going through the fully connected layer in the last layer. Hence, the duration of time for the convolution neural network model to learn the image data set much faster than fully connected layer.

There were two solutions that were being used in solving overfitting issues - by adjusting the dropout value and to stop the epochs when the training and validation accuracy start to over fit. Table 6 show the training accuracy and testing accuracy for each dropout and epochs training for fully connected layer and convolution neural network model. From Table 6 , suitable dropout value of 0.2 for fully connected layer and 0.5 for convolution neural network was found to be the most optimum setting to produce result with desired accuracy. 


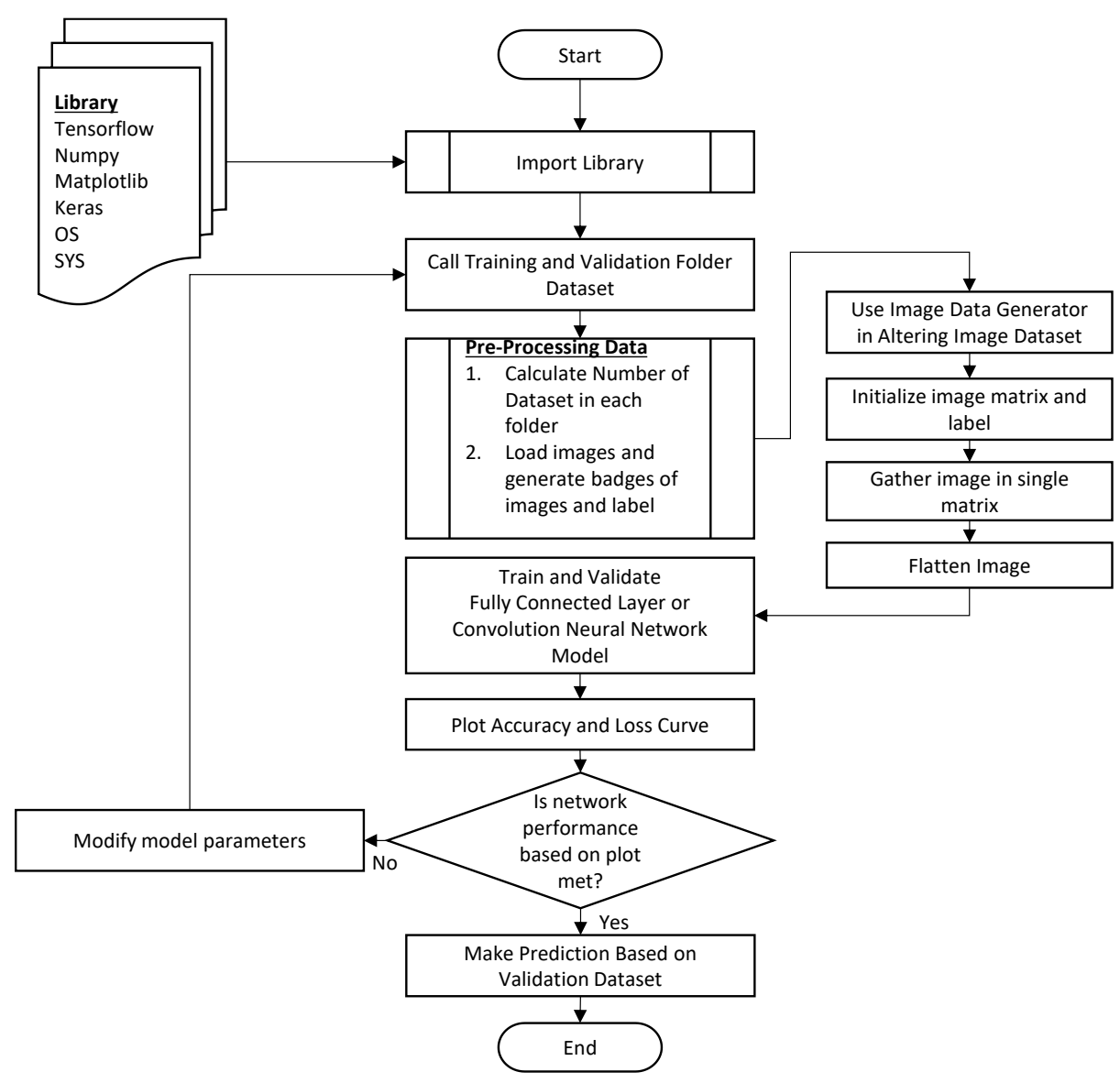

Figure 3: Flow chart for Deep learning algorithm of fully connected layer model or convolution neural network model

Once the most optimum dropout value is identified to match the needed accuracy, the available image in testing dataset is tested. Next, prediction is made using the slope images captured around the IIUM. Fig. 5 shows the image data set tested for fully connected layer and convolution neural network. These slope images are classified as high risk by manual calculation assisted by local expert. Comparing the two methods, prediction using convolution neural network shows a higher accuracy compared to fully connected layer. In a fully connected layer, out of 12 high risk images, only 4 images or $33 \%$ are labeled correctly, while the rest are labelled as medium (6 images) and low (2 images). Comparatively, in a convolution neural network, using the sample 12 images sampled, 8 out of 12 images are predicted correctly, an accuracy of $67 \%$. Most importantly, the other 4 are labeled a medium risk. Thus, making convolution neural network is able to give a better prediction, with lower risk of false negative. Based on data above and plot in Figure 3, it is observed that overfitting error is lowest in a convolution neural network around $67 \%$ testing accuracy on 0.5 dropout values. The same dropout value was being used in making prediction on slope around IIUM area.

Using a mobile phone camera, 12 images of slope around IIUM was captured. These images will be utilized to determine the hazard of the slope within IIUM area. These images had been captured in differences location within the campus - Faculty of Information Communication Technology, Stadium and Salahuddin Hostel as shown in Fig. 6. All the images captured within the campus are labelled as low risk by the local expert. Using the convolution neural network prediction model having $67 \%$ testing accuracy, it was found that 6 images were labelled correctly as low risk, while the other 6 images have been labelled with false positive of high risk. This makes the accuracy of the convolution neural network prediction having difference of $17 \%$ between the predicted accuracy from testing sample images at $67 \%$ 
and the actual accuracy using IIUM images at 50\%, with summary of the results show in Table 7 . To improve the prediction value, additional data need to be used to train the convolution neural network.

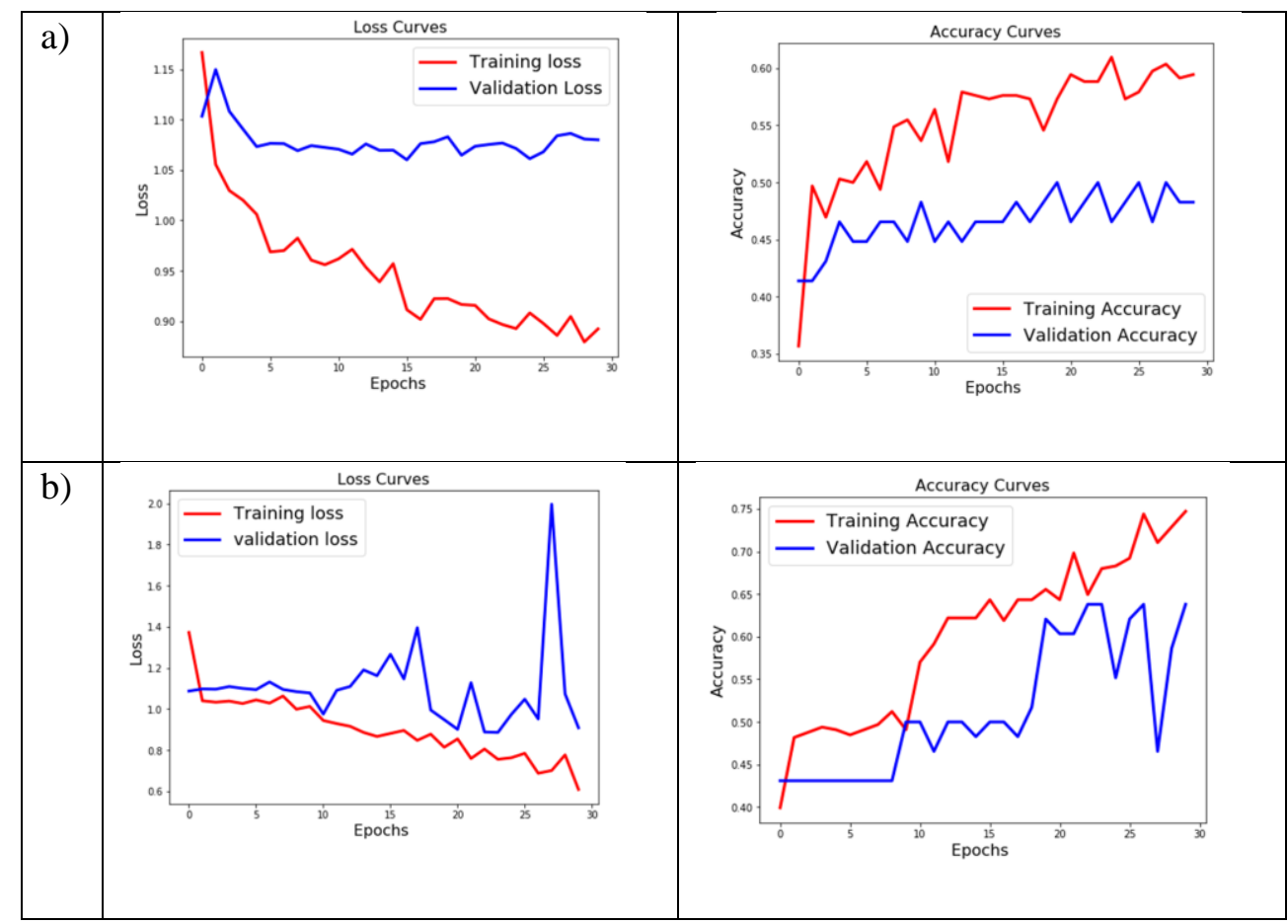

Figure 4: a) Loss curves and accuracy curves for fully connected layer b) Loss curves and accuracy curves for convolution neural network model.

Table 6: Training accuracy and testing accuracy for different dropout value in fully connected layer model and CNN model

\begin{tabular}{|c|c|c|c|c|c|}
\hline \multirow{2}{*}{$\begin{array}{c}\text { Dropout } \\
\text { value }\end{array}$} & \multirow{2}{*}{ Epochs } & \multicolumn{2}{|c|}{ Fully Connected Layer } & \multicolumn{2}{c|}{$\begin{array}{c}\text { Convolution Neural } \\
\text { Network }\end{array}$} \\
\cline { 3 - 6 } & & $\begin{array}{c}\text { Training } \\
\text { Accuracy }\end{array}$ & $\begin{array}{c}\text { Testing } \\
\text { Accuracy }\end{array}$ & $\begin{array}{c}\text { Training } \\
\text { Accuracy }\end{array}$ & $\begin{array}{c}\text { Testing } \\
\text { Accuracy }\end{array}$ \\
\hline 0 & 30 & 0.46197182 & 0.43396226 & 0.909859 & 0.566037 \\
\hline 0.2 & 30 & 0.5380282 & 0.43396226 & 0.867605 & 0.698113 \\
\hline 0.3 & 30 & 0.49859154 & 0.4528302 & 0.681690 & 0.584905 \\
\hline 0.4 & 30 & 0.44225353 & 0.3773585 & 0.726760 & 0.547169 \\
\hline 0.5 & 30 & 0.46197182 & 0.43396226 & 0.630985 & 0.6792453 \\
\hline
\end{tabular}


Table 7: Summary of Actual Accuracy Using Sampled Images and Actual IIUM Images

\begin{tabular}{|c|c|c|c|c|}
\hline \multirow{2}{*}{\multicolumn{2}{|c|}{ Details }} & \multicolumn{2}{|c|}{ Sampled Images } & \multirow{2}{*}{$\begin{array}{l}\text { Actual IIUM } \\
\text { Images }\end{array}$} \\
\hline & & $\begin{array}{l}\text { Fully Connected } \\
\text { Later }\end{array}$ & $\begin{array}{c}\text { Convolution } \\
\text { Neural Network }\end{array}$ & \\
\hline \multicolumn{2}{|c|}{ Total No. of Images } & 12 & 12 & 12 \\
\hline \multicolumn{2}{|c|}{ Risk Assessed by Local Expert } & All High & All High & All Low \\
\hline \multirow{3}{*}{$\begin{array}{l}\text { No. of Images by Risk } \\
\text { Level, Predicted by } \\
\text { Network }\end{array}$} & Low & 2 & 0 & 6 \\
\hline & Medium & 6 & 4 & 0 \\
\hline & High & 4 & 8 & 6 \\
\hline \multicolumn{2}{|c|}{$\begin{array}{l}\text { Actual Accuracy Compared to } \\
\text { Local Expert. }\end{array}$} & $33 \%$ & $67 \%$ & $50 \%$ \\
\hline
\end{tabular}
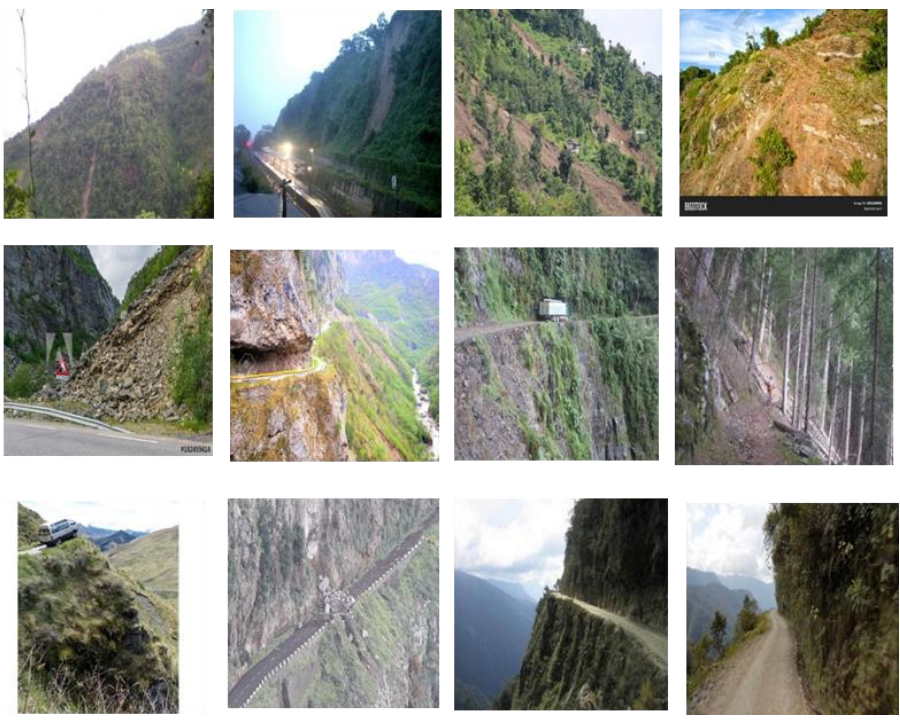

Figure 5: Images being used in testing the prediction result.
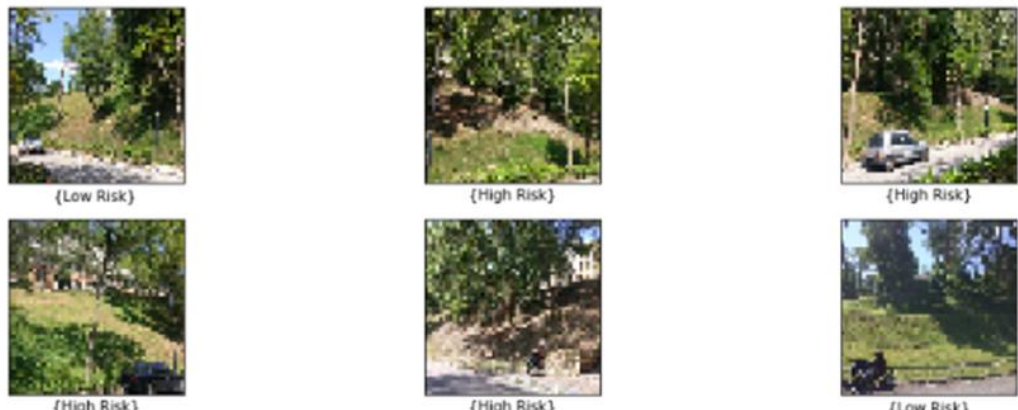

[High Risk]

\{High Risk\}
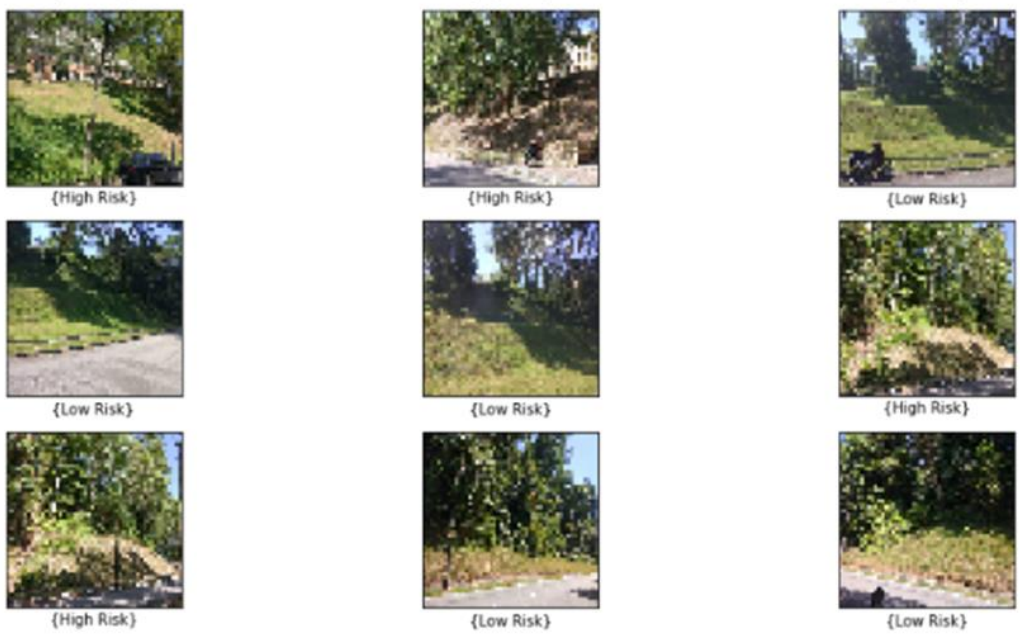

Figure 6: The result of prediction of the risk analysis for IIUM area. 


\section{CONCLUSION}

In this study a method to identify the risk level of a slope have been fully identified and develop. However, the accuracy afforded by the system still need to be improved further. With accuracy of actual image CNN prediction reduced by $17 \%$ compared to trained $\mathrm{CNN}$ prediction, additional optimization and work is needed to ensure that slope imaging technique could be utilized mainstream. It is recommended that more different variation of images to be captured, labelled and trained by relevant local experts to improve the overall performance of the system.

\section{REFERENCE}

[1] N. Makaremi, E. Salleh, M. Z. Jaafar, and A. GhaffarianHoseini, 'Thermal comfort conditions of shaded outdoor spaces in hot and humid climate of Malaysia', Building and Environment, vol. 48, pp. 7-14, Feb. 2012, doi: 10.1016/j.buildenv.2011.07.024.

[2] S. Leoi, A. Chan, and N. Trisha, 'Malaysia among countries especially prone to landslides | The Star'. https://www.thestar.com.my/news/nation/2018/12/04/msia-ranks-highly-for-landslidescountry-experienced-185-occurrences-annually-in-past-10-years/ (accessed Aug. 27, 2021).

[3] H. Abdul Rahman and J. Mapjabil, 'Landslides Disaster in Malaysia: an Overview', Health and the Environment Journal, vol. 8, pp. 58-71, Jan. 2017.

[4] T. B. Afeni and F. T. Cawood, 'Slope Monitoring using Total Station: What are the Challenges and How Should These be Mitigated?', South African Journal of Geomatics, vol. 2, no. 1, Art. no. 1, Jan. 2013.

[5] X. Hou, 'Geotechnical Engineering Slope Monitoring Based on Internet of Things', International Journal of Online and Biomedical Engineering (iJOE), vol. 14, no. 06, Art. no. 06, Jun. 2018.

[6] S. A. K. Tareen and H. M. Khan, 'Novel slope detection and calculation techniques for mobile robots', in 2016 2nd International Conference on Robotics and Artificial Intelligence (ICRAI), Nov. 2016, pp. 158-163. doi: 10.1109/ICRAI.2016.7791246.

[7] N. Anwar, F. Najam, and M. Amir Izhar, 'Construction Monitoring and Reporting using Drones and Unmanned Aerial Vehicles (UAVs)', presented at the The Tenth International Conference on Construction in the 21st Century (CITC-10), Colombo, Sri Lanka, Jul. 2018.

[8] I. Jaukovic and A. Hunter, 'Unmanned Aerial Vehicles: A new tool for landslide risk assessment', presented at the 11th ANZ Young Geotechnical Professionals Conference, Queenstown, New Zealand, Oct. 2016.

[9] W. Frodella, G. Gigli, S. Morelli, L. Lombardi, and N. Casagli, 'Landslide Mapping and Characterization through Infrared Thermography (IRT): Suggestions for a Methodological Approach from Some Case Studies', Remote Sensing, vol. 9, no. 12, p. 1281, Dec. 2017, doi: 10.3390/rs9121281.

[10] M. van Veen, M. Lato, D. Hutchinson, and R. Kromer, 'The Role of Survey Design in Developing Rock Fall Frequency-Magnitude Relationships using Terrestrial Laser Scanning: A Case Study from the CN Railway at White Canyon, BC', presented at the 3rd North American Symposium on Landslides, Jun. 2017.

[11] A. Pepe and F. Calò, 'A Review of Interferometric Synthetic Aperture RADAR (InSAR) MultiTrack Approaches for the Retrieval of Earth's Surface Displacements', Applied Sciences, vol. 7, no. 12, Art. no. 12, Dec. 2017, doi: 10.3390/app7121264.

[12] O. Ghorbanzadeh, T. Blaschke, K. Gholamnia, S. R. Meena, D. Tiede, and J. Aryal, 'Evaluation of Different Machine Learning Methods and Deep-Learning Convolutional Neural Networks for Landslide Detection', Remote Sensing, vol. 11, no. 2, Art. no. 2, Jan. 2019, doi: 10.3390/rs11020196.

[13] B. Feizizadeh, M. Shadman Roodposhti, P. Jankowski, and T. Blaschke, 'A GIS-based extended fuzzy multi-criteria evaluation for landslide susceptibility mapping', Computers \& Geosciences, vol. 73, pp. 208-221, Dec. 2014, doi: 10.1016/j.cageo.2014.08.001. 
[14] M. R. Mezaal, B. Pradhan, M. I. Sameen, H. Z. Mohd Shafri, and Z. M. Yusoff, 'Optimized Neural Architecture for Automatic Landslide Detection from High-Resolution Airborne Laser Scanning Data', Applied Sciences, vol. 7, no. 7, Art. no. 7, Jul. 2017, doi: 10.3390/app7070730.

[15] G. Danneels, E. Pirard, and H.-B. Havenith, 'Automatic landslide detection from remote sensing images using supervised classification methods', in 2007 IEEE International Geoscience and Remote Sensing Symposium, Jul. 2007, pp. 3014-3017. doi: 10.1109/IGARSS.2007.4423479.

[16] X. X. Zhu et al., 'Deep Learning in Remote Sensing: A Comprehensive Review and List of Resources', IEEE Geoscience and Remote Sensing Magazine, vol. 5, no. 4, pp. 8-36, Dec. 2017, doi: 10.1109/MGRS.2017.2762307.

[17] F. C. Dai, C. F. Lee, and Y. Y. Ngai, 'Landslide risk assessment and management: an overview', Engineering Geology, vol. 64, no. 1, pp. 65-87, Apr. 2002, doi: 10.1016/S0013-7952(01)00093$\mathrm{X}$.

[18] S. Jamaludin and A. N. Hussein, 'Landslide hazard and risk assessment: The Malaysian experience', presented at the 10th Congress of the International Association for Engineering Geology and the Environment (IAEG), London, United Kingdom, Jul. 2006.

[19] W. S. Chow and Z. Mohamad, 'Terrain and landslide hazards mapping for disaster management', Department of Mineral and Geoscience Malaysia., Internal Unpublished, 2002.

[20] J. Ab. Talib, 'Landslide hazard zonation mapping of Selangor', Bulletin of the Institution of Engineers Malaysia, vol. 10, no. 1, 2001.

[21] RafieTarabay, 'Deep Learning', IBM Developer Recipes, Oct. 31, 2019. https://developer.ibm.com/recipes/tutorials/deep-learning/ (accessed Aug. 28, 2021). 